\title{
TOMADORES DE DECISIÓN EN SISTEMAS GANADEROS DE LA CUENCA ALTA DEL RÍO GUARINÓ (CALDAS, COLOMBIA): PERCEPCIÓN DE PROBLEMAS AMBIENTALES Y PRÁCTICAS DE CONSERVACIÓN DEL AGUA
}

\author{
MARCELA PATIÑO MURILLO ${ }^{1}$ \\ ISAÍAS TOBASURA ACUÑA² \\ isaias.tobasura@ucaldas.edu.co
}

Manizales, 2011-04-30 (Rev. 2011-10-24)

\section{RESUMEN}

En la conservación de los recursos naturales y la preservación de la calidad ambiental es muy importante la participación de los tomadores de decisión, sobre todo de los productores locales. Por ello, en este artículo se presentan algunas características de tomadores de decisión en predios de sistemas ganaderos de la cuenca alta del río Guarinó. Entre ellas se destacan: género, origen, tenencia y tamaño del predio, edad, educación, participación, entre otros. El estudio permitió evidenciar la heterogeneidad de los ganaderos de la zona, y reconocer los problemas ambientales que perciben y las prácticas de conservación del agua que usan. Y aunque no se encontraron relaciones significativas entre las características de los tomadores de decisión, los problemas ambientales y las prácticas de conservación, con base en los resultados se hacen algunas recomendaciones para el diseño de estrategias de conservación en la cuenca.

\section{PALABRAS CLAVE:}

Decisiones, ganadería, cuenca, ambiente, participación.

\section{DECISION MAKING ACTORS IN LIVESTOCK SYSTEMS IN THE HIGH GUARINÓ RIVER BASIN (CALDAS, COLOMBIA): PERCEPTION OF ENVIRONMENTAL PROBLEMS AND WATER CONSERVATION PRACTICES.}

\begin{abstract}
In the conservation of natural resources and the preservation of environmental quality, the participation of the decision making actors, specially the local producers, is very important,. This way, some characteristics of the decision making actors in livestock systems in the high Guarinó river basin are presented in this article. Some of them highlight: gender, origin, piece of land ownership and size, age, education, participation, among others. This study allowed to evidence the heterogeneity of livestock farmers in the region, and to recognize, the environmental problems they perceive and the water conservation practices they use. And even though significant relations between characteristics of the decision making actors, the environmental problems and the conservation practices were not found, some recommendations for the design of conservation strategies in the high basin are made based upon the results found.
\end{abstract}

\section{KEY WORDS:}

Decisions, livestock, basin, environment, participation 


\section{INTRODUCCIÓN}

La actividad ganadera provee medios de sustento para la mayor parte de la población rural en el mundo (FAO, 2007), y en Colombia genera $25 \%$ de empleos rurales, siendo desarrollada principalmente por pequeños y medianos productores (FEDEGAN, 2006), pertenecientes a diversos grupos sociales y étnicos (Murgueitio, 1999). Sin embargo, el desarrollo de esta actividad genera impactos ambientales negativos reconocidos mundialmente (Steinfeld et al., 2006) y documentados en el país, como deforestación, erosión, pérdida de biodiversidad e impactos sobre el agua (Murgueitio, 2003).

Actualmente en el país los temas ambientales relacionados con la ganadería son parte de la planeación del sector, donde existen metas concretas para alcanzar en el año 2019, entre ellas, devolverle a la naturaleza 10 millones de hectáreas que sin ser aptas para la actividad ganadera están dedicadas a esta (FEDEGAN, 2006).

En los procesos de conservación ambiental y el manejo integrado de los recursos naturales es necesario incorporar a los principales responsables del manejo de los recursos, los actores locales, ya que el concepto de ecosistema o ambiente natural, lo mismo que la valoración que se le asigne, depende de los beneficios que cada actor obtiene y de las características de estos actores, como la diversidad cultural, el nivel de educación, el nivel socioeconómico, entre otros (Delgado, Bachmann \& Oñate, 2007).

Las acciones ambientales, deben considerar las características de los productores agropecuarios y tener en cuenta las condiciones biofísicas de los predios. Es decir, consultar las realidades locales (Gil et al., 2008) y, específicamente, para el establecimiento de sistemas ganaderos que busquen hacer compatible el desarrollo socioeconómico con la protección de la naturaleza, es indispensable tanto la identificación de los actores involucrados en la toma de decisiones, como de la diversidad de situaciones en que se realiza esta actividad y los impactos socialesambientales que genera (Murgueitio, 1999).

Entendida la percepción como la visión personal de los actores locales hacia situaciones particulares (Nieto, Riedel \& Vera, 2002), la percepción sobre la degradación ambiental es importante de entender, entre otras razones porque: 1) sirve como un indicador útil y objetivo de degradación ambiental (cuando no hay otros datos disponibles), 2) influye en la participación de los actores locales en programas y políticas dirigidas a mejorar las condiciones ambientales, y 3 ) influye en los comportamientos ambientales individuales (Barber, Biddlecom \& Axinn, 2003).

Para revertir la tendencia actual del agotamiento de los recursos naturales que produce la actividad ganadera, existen múltiples opciones de mitigación que se basan en tres principios fundamentales: 1) el uso más reducido de los recursos, 2) un menor agotamiento y 3) una mayor reposición de los recursos (Steinfeld et al., 2006; FAO, 2007). En el país, se han propuesto muchas prácticas y estrategias de conservación de recursos naturales en los sistemas ganaderos (Murgueitio et al., 2008).

En este orden de ideas, una estrategia de conservación, para que tenga un impacto real en el tiempo, debe incorporar a las sociedades humanas en el manejo de los recursos naturales, considerar sus percepciones sobre realidades locales e identificar las situaciones particulares que generan o mitigan impactos ambientales. De esa manera, se lograrán 
transformar actividades como la ganadera que, por sus características, genera externalidades negativas en los recursos naturales y el medio ambiente.

En la cuenca alta del Río Guarinó la ganadería es la principal actividad económica. Los impactos de esta actividad en el medio ambiente han sido documentados (CORPOCALDAS, 2001) y actualmente existe interés por conservar la cuenca. Esta situación ha generado algunas iniciativas de conservación, realizadas en conjunto con la Corporación Autónoma Regional de Caldas (CORPOCALDAS), el municipio de Marulanda y algunos ganaderos de la zona, entre ellas acciones de reforestación y de promoción de la agroforestería, cercas vivas, arreglos silvopastoriles y conservación de las fuentes de agua.

Mediante análisis de estadística descriptiva, en este trabajo se buscó generar información de base que permitiera tener una visión regional de la cuenca alta del río Guarinó, particularmente en cuatro aspectos: 1) Características de los tomadores de decisión ${ }^{3}$ a nivel de predios ganaderos, 2) Percepción que estos tienen de los problemas ambientales en la zona, 3) Acciones de conservación desde los predios ganaderos, 4) Relaciones entre las características de los tomadores de decisión, la percepción de los problemas ambientales y las prácticas de conservación implementadas en la zona.

Esta información es fundamental para el diseño de programas de manejo de los sistemas ganaderos, que involucre, además de los productores, otros tomadores de decisión como son las autoridades ambientales, los administradores municipales, y las entidades relacionadas o interesadas en la conservación de la cuenca.

\section{MATERIALES Y MÉTODOS}

Zona de estudio, muestra y recolección de datos

La zona de estudio se ubica en la región alto andina de Colombia a 5017'3" latitud norte y 74015'48" longitud occidental, sobre la Cordillera Central en jurisdicción del municipio de Marulanda (Caldas) (Figura 1). Las condiciones agroecológicas de la zona son de relieve montañoso, pendientes moderadas y altas, clima promedio de $140 \mathrm{C}$, altura promedio de $3100 \mathrm{msnm}$, en ecosistema de bosque alto andino, caracterizado por su amplia diversidad biológica (Parques Nacionales Naturales de Colombia, s.f.).

La hidrografía de la zona pertenece a la vertiente oriental de la Cordillera Central, con una red de drenajes que fluyen en sentido oeste-este, y desembocan en el cauce del río Guarinó, que nace en la vereda El Páramo y recorre el municipio de noroccidente hasta suroriente, y recibe a su paso aguas de afluentes y continúa por el límite departamental hasta desembocar en la cuenca del río Magdalena. El área aproximada de toda la cuenca del río Guarinó es de $624,85 \mathrm{~km}^{2}, 57 \%$ pertenece al municipio de Marulanda (CORPOCALDAS, 2001). En relación a la cuenca alta y sus principales afluentes (Figura 1), se ubican aproximadamente 120 predios principalmente ganaderos, cuyo objetivo productivo es la comercialización de leche, queso y carne en mercados locales y regionales. 
Figura 1. Ubicación general de la zona y predios incluidos en el estudio en el municipio de Marulanda (Caldas, Colombia).
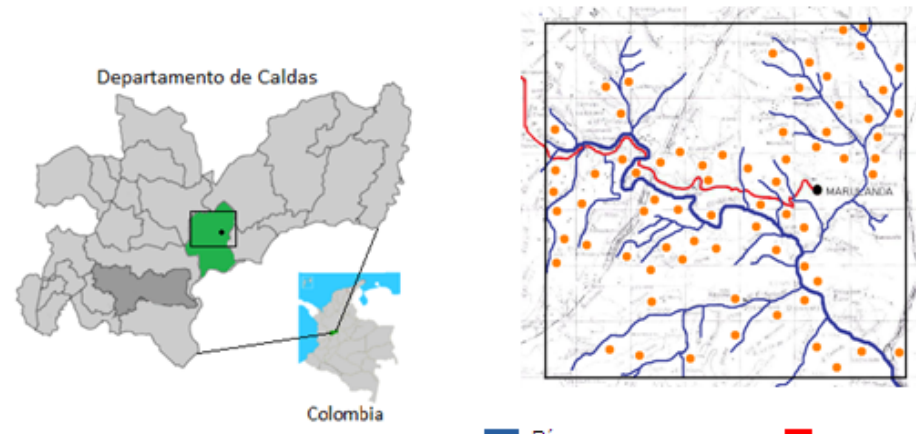

Para la muestra se escogieron por selección aleatoria simple (Berenson \& Levine, 1991), un total de 69 predios ganaderos del sector, con un nivel de confianza de $93 \%$, y un error de $7 \%$. Para la recolección de datos se aplicó una encuesta dirigida a los tomadores de decisión de los sistemas productivos. Esta encuesta se aplicó entre los meses de febrero-abril de 2009, previo contacto telefónico con el encuestado y en diferentes sitios, ya fuera en el predio, sitios de trabajo o sitios de acopio. Dentro de la encuesta se incluyeron preguntas dirigidas a recolectar información sobre: 1) Información básica del predio, 2) Información del tomador de decisiones, 3) Información ambiental, 4) Información complementaria. Para la tabulación de los datos y análisis estadísticos se utilizó Excel 2007.

\section{RESULTADOS Y DISCUSIÓN}

\section{Tomadores de decisión en predios}

Los resultados de las características de los tomadores de decisión en los predios ganaderos se presentan en el Cuadro 1, y a continuación se describen las principales observaciones. 
Cuadro 1. Características de los tomadores de decisión en predios ganaderos de la cuenca alta del río Guarinó.

\begin{tabular}{|c|c|c|c|}
\hline \multicolumn{2}{|c|}{ Características } & No. de predios & $\%$ \\
\hline \multirow[t]{2}{*}{ Cantidad } & 1 & 59 & 85 \\
\hline & Más de 1 & 10 & 15 \\
\hline \multirow{2}{*}{ Género } & Hombre & 67 & 97 \\
\hline & Mujer & 2 & 3 \\
\hline \multirow{3}{*}{ Tamaño* } & Pequeños & 13 & 19 \\
\hline & Medianos & 54 & 78 \\
\hline & Grandes & 2 & 3 \\
\hline \multirow{3}{*}{ Origen } & Municipio & 40 & 58 \\
\hline & Departamento & 23 & 33 \\
\hline & Otro departamento & 6 & 9 \\
\hline \multirow{3}{*}{ Tenencia } & Propio & 59 & 86 \\
\hline & Arriendo & 9 & 13 \\
\hline & Otro & 1 & 1 \\
\hline \multirow[t]{2}{*}{ Ocupación } & Habita en predio & 16 & 23 \\
\hline & No habita en predio & 53 & 77 \\
\hline \multirow{5}{*}{ Edad } & 13-30 años & 3 & 4 \\
\hline & $31-45$ & 28 & 41 \\
\hline & $46-60$ & 20 & 29 \\
\hline & $61-75$ & 14 & 20 \\
\hline & $76-90$ & 4 & 6 \\
\hline \multirow{8}{*}{ Educación } & Ningún estudio & 1 & 1,5 \\
\hline & Primaria incompleta & 10 & 14,5 \\
\hline & Primaria completa & 21 & 30 \\
\hline & Bachillerato incompleto & 10 & 14,5 \\
\hline & Bachillerato completo & 9 & 13 \\
\hline & Técnica completa & 6 & 9 \\
\hline & Universitaria incompleta & 1 & 1,5 \\
\hline & Universitaria completa & 11 & 16 \\
\hline \multirow{2}{*}{ Participación } & $\mathrm{Si}$ & 45 & 65 \\
\hline & No & 24 & 35 \\
\hline \multirow{2}{*}{ Capacitación } & $\mathrm{Si}$ & 35 & 51 \\
\hline & No & 34 & 49 \\
\hline Asistencia técnica & $\begin{array}{l}\text { Si } \\
\text { No }\end{array}$ & $\begin{array}{l}17 \\
52\end{array}$ & $\begin{array}{l}25 \\
75\end{array}$ \\
\hline
\end{tabular}

*Utilizando como referencia el número de bovinos en el predio: Pequeños (hasta 50 animales), Medianos (51 a 500 animales) y Grandes (501 o más animales) (FEDEGAN, 2006).

Cantidad. En la mayoría de los predios solo una persona toma las decisiones, sin embargo, en algunos las decisiones se toman de manera conjunta. De los predios encuestados, en 59 (85\%) solo una persona toma las decisiones mientras que en $10(15 \%)$ son dos personas quienes toman las decisiones, en estos últimos, en 5 casos el propietario del predio y alguno de sus hijos, y en 5 casos el propietario y un administrador contratado ${ }^{4}$.

Género. En la mayoría de predios los tomadores de decisión son hombres. En 67 de los predios encuestados (97\%) las decisiones son tomadas por hombres, mientras que solo en 2 predios (3\%) las decisiones son tomadas por mujeres, que son cabeza de hogar. 
Tamaño. Según la clasificación de FEDEGAN, podemos inferir que en la cuenca alta del río Guarinó la mayoría de los tomadores de decisión son medianos productores con un total de $54(78 \%)$, seguidos por pequeños con $13(19 \%)$ y con solo $2(3 \%)$ correspondiente a grandes.

Origen. En cuanto al origen de los tomadores de decisión, la mayoría son nacidos en el municipio. 40 entrevistados (58\%) son nacidos en Marulanda, 23 (33\%) son nacidos en otros municipios del departamento y $6(9 \%)$ son nacidos en otros departamentos del país.

Tenencia y ocupación del predio. En la zona, los tomadores de decisión en su mayoría son propietarios del predio pero no habitan en él. De los encuestados $59(86 \%)$ son propietarios, $9(13 \%)$ arriendan el predio y solo en un predio se encontró tenencia incierta (en proceso de sucesión). Sin embargo, de los encuestados, solo $16(23 \%)$ viven en el predio, mientras que $53(77 \%)$ no habitan en él.

Edad. Las edades de los tomadores de decisión son variables, y oscilan entre 26 y 85 años cumplidos. La mayoría de los tomadores de decisión se encuentran en el rango de 31 a 45 años de edad con 28 individuos (41\%), seguidos por edades entre 46 y 60 años con 20 individuos (29\%). Es de anotar que solo en 3 predios (4\%) los tomadores de decisión están en el rango de 16 a 30 años cumplidos.

Educación. El nivel de educación de los tomadores de decisión en la zona es relativamente alto, aunque es importante considerar las diferencias existentes. La mayoría de los encuestados, en total 21, tienen primaria completa $(30 \%)$, seguidos por 11 tomadores de decisión con estudios universitarios completos (16\%), 10 con bachillerato incompleto $(14,5 \%), 9$ con bachillerato completo (13\%), 6 con estudios técnicos completos $(9 \%)$ y 1 con estudios universitarios incompletos; estas cifras en general hablan de un buen nivel de educación. Sin embargo, existen 11 tomadores de decisión (16\%) que no tienen ningún tipo de estudio o solo tienen estudios de primaria incompleta.

Participación. Con relación a la participación en diferentes organizaciones de la región, se encontró que la mayoría de tomadores de decisión participan en algún tipo de organización local-regional, la mayoría en la Cooperativa Ovina. En total $45(65 \%)$ participan, mientras que 24 (35\%) no lo hacen.

Capacitación. De los entrevistados, 35 (51\%) han recibido o han asistido a algún tipo de capacitación, mientras que $34(49 \%)$ no lo han hecho. Las capacitaciones que han recibido los productores, en su mayoría, han sido de temas productivos $(77 \%)$.

Uso de asistencia técnica. La asistencia técnica que utilizan los tomadores de decisión en la zona es baja. En 17 predios (25\%) los tomadores de decisión usan asistencia técnica, mientras que en 52 predios $(75 \%)$ no la usan.

Con base en los resultados arriba descritos, se puede afirmar que en la cuenca alta del río Guarinó, los predios ganaderos son administrados en su mayoría por una sola persona, por lo general hombre, nacido en la región, propietario de la tierra aunque no habita en ella y con frecuencia participante en alguna organización local o regional. Por el tamaño de las explotaciones que manejan, la mayoría de los tomadores de decisión son medianos con 51 a 500 animales y pequeños con hasta 50 animales. 
Entre los tomadores de decisión de la zona se evidencian edades y niveles de educación variados, confiriendo sobre estos características de heterogeneidad notables. En este sentido puede decirse que la zona es representativa de la heterogeneidad característica de los sistemas ganaderos del país, como sucede en la ganadería de la región andina, donde en todo tipo de terrenos y predios, con tamaños desde una o dos hectáreas, hasta 500 hectáreas, se encuentran empresarios agropecuarios de sistemas exclusivamente ganaderos o mixtos y también campesinos, indígenas o colonos (Murgueitio, 2003).

En cuanto a otros aspectos de los tomadores de decisión como capacitación, asistencia técnica, se encontró que aproximadamente la mitad fueron capacitados y la mayoría no usa asistencia técnica, características que podrían constituirse como debilidades para la gestión y manejo de los predios.

Esta caracterización debe ser tomada en cuenta en el momento de realizar intervenciones, acciones o proyectos dirigidos a la conservación de la cuenca. Reconocer las particularidades de los tomadores de decisión en la zona y las variaciones que estos presentan en cuanto al tamaño de los predios que manejan, la edad y el nivel educativo que poseen. Además, en lo posible involucrar en la toma de decisiones de los sistemas productivos, sobre todo a las mujeres.

\section{Percepción de problemas ambientales}

Con relación a los problemas ambientales que perciben los tomadores de decisión en la zona, se evidencia que los cambios del clima son el problema percibido con mayor frecuencia (94\%), seguido por los problemas de erosión (30\%), la disminución en la cantidad/calidad del agua $(26 \%)$, los deslizamientos $(19 \%)$ y la deforestación (17\%). La pérdida de biodiversidad es el problema menos percibido por los tomadores de decisión de los diferentes predios ganaderos (16\%) (Cuadro 2). Esta diferencia en la percepción de los problemas ambientales, podría explicarse porque los problemas más percibidos se relacionan directamente con los sistemas de producción y sus rendimientos, mientras que los menos percibidos no tienen relación directa con los sistemas de producción o sus rendimientos.

Cuadro 2. Problemas ambientales percibidos por los tomadores de decisión de predios ganaderos.

\begin{tabular}{lll}
\hline Problema ambientales & Frecuencia percepción & $\%^{*}$ \\
\hline Cambios del clima & 65 & 94 \\
Erosión & 21 & 30 \\
Cantidad/calidad del agua & 18 & 26 \\
Deslizamientos & 13 & 19 \\
Deforestación & 12 & 17 \\
Pérdida de biodiversidad & 11 & 16 \\
No percibe problemas & 1 & 1 \\
\hline${ }^{*}$ Sobre el total de predios en el estudio (69).
\end{tabular}

Considerando que la percepción de los problemas ambientales es conducente a promover la participación de las personas y a cambiar las actitudes individuales frente a estos (Barber, Biddlecom \& Axinn, 2003), se podría esperar que en la zona de estudio las acciones de conservación que conduzcan a mitigar las problemáticas más percibidas, como cambio del clima, erosión y calidad/cantidad del agua sean bien acogidas, mientras que las acciones que conduzcan a mitigar las problemáticas menos percibidas, como deforestación y pérdida de biodiversidad, no 
tengan buena acogida. En este sentido, los problemas menos percibidos necesitan entrar en la "conciencia" de los tomadores de decisión, por ejemplo, a través de capacitaciones que ilustren su importancia.

\section{Prácticas de conservación del agua}

Para facilitar la presentación de las prácticas encontradas y utilizadas por los ganaderos, utilizamos los principios que propone la FAO (2007) para clasificar las opciones de mitigación sobre los recursos hídricos. De esta manera, las prácticas de conservación del agua utilizadas en la zona de estudio se clasifican en tres tipos (Cuadro 3).

Los resultados de las encuestas evidencian que en la mayoría de los predios de la cuenca se implementan prácticas de conservación dirigidas a la conservación del agua. En 53 predios (76\%) se utiliza alguna práctica de conservación, mientras que en $16(23 \%)$ no se utiliza ninguna. Probablemente, esto obedezca a que no exista la percepción de la necesidad de hacerlo, debido a que las fuentes de agua están bien conservadas.

Cuadro 3. Clasificación de las prácticas de conservación del agua implementadas en la zona de estudio según principios de la FAO (2007).

\begin{tabular}{ll}
\hline TIPOS DE PRACTICAS (FAO, 2007) & PRACTICAS EN ZONA DE ESTUDIO \\
\hline $\begin{array}{l}\text { Tipo 1: Para uso más reducido del } \\
\text { agua. }\end{array}$ & $\begin{array}{l}\text { Uso de flotadores en tanques. } \\
\text { suministro. } \\
\text { Uso de contadores de agua. }\end{array}$ \\
\hline Tipo 2: Para disminuir el agotamiento. & $\begin{array}{l}\text { Uso controlado de pesticidas. } \\
\text { Uso de compostaje (como abono). }\end{array}$ \\
\hline $\begin{array}{l}\text { Tipo 3: Para mayor reposición del } \\
\text { agua. }\end{array}$ & $\begin{array}{l}\text { Delimitación de zonas críticas para el agua. } \\
\text { Reforestación de zonas críticas para el } \\
\text { agua. }\end{array}$ \\
\end{tabular}

De acuerdo a esta clasificación y analizadas de forma individual, las prácticas más utilizadas para la conservación del agua en la zona, son las de Tipo 3 usadas en 45 predios (84\%), seguidas por las de Tipo 1 usadas en 41 predios $(77 \%)$. Las prácticas menos utilizadas en la zona son las de Tipo 2, solo en 4 predios ( $8 \%$ ) (Cuadro 4).

Cuadro 4. Uso de los 3 tipos de prácticas de conservación del agua en la zona de estudio (por orden de frecuencia).

\begin{tabular}{ccc}
\hline $\begin{array}{c}\text { Tipo de prácticas } \\
\text { individuales }\end{array}$ & Frecuencia & $\%^{*}$ \\
\hline Tipo 3 & 45 & 84 \\
Tipo 1 & 41 & 77 \\
Tipo 2 & 4 & 8 \\
\hline
\end{tabular}

"Sobre el total de predios en que se usan prácticas de conservación (53).

Al analizar los 69 predios considerados en el estudio y el uso de prácticas de conservación del agua, es evidente que existen usos individuales de las prácticas, combinaciones de uso o ausencia de prácticas. En el Cuadro 5 se observa que 29 de los predios (42\%) utilizan las prácticas 
Tipo 1 junto con las Tipo 3, seguidos en frecuencia por los predios donde no se utiliza ningún tipo de práctica: $16(23 \%)$. En 8 predios $(12 \%)$ se utilizan solo prácticas Tipo 1, y en 2 predios (3\%) solo prácticas Tipo 2. Según los resultados, solo en 4 predios $(6 \%)$ se utilizan los 3 tipos de prácticas. Estos resultados indican que en cuanto a los tres tipos de prácticas, estas se implementan de maneja conjunta o individual, siendo la combinación más utilizada el uso de las Tipo 1 en conjunto con las Tipo 3.

Cuadro 5. Uso de prácticas de conservación del agua en predios ganaderos de la cuenca (por orden de frecuencia).

\begin{tabular}{lcc}
\hline $\begin{array}{c}\text { Uso de prácticas individuales } \\
\text { o combinadas en predios }\end{array}$ & Frecuencia & $\%{ }^{*}$ \\
\hline Tipo 1 + Tipo 3 & 29 & 42 \\
Ninguna & 16 & 23 \\
Tipo 3 & 10 & 14 \\
Tipo 1 & 8 & 12 \\
Tipo 1 + Tipo 2 + Tipo 3 & 4 & 6 \\
Tipo 2 & 2 & 3 \\
\hline "Sobre el total de predios en el estudio (69).
\end{tabular}

Relación entre características de los tomadores de decisión y la percepción de problemas ambientales

Este análisis no muestra resultados significativos, sin embargo, es evidente por la tendencia de los signos que existen relaciones directas entre la percepción de problemas ambientales y algunas características de los tomadores de decisión como: el tamaño $(+)$, la edad $(+)$ y la capacitación (+) (Cuadro 6).

Cuadro 6. Coeficientes de Correlación entre problemas ambientales percibidos y características de los tomadores de decisión de los predios ganaderos de la cuenca.

\begin{tabular}{lccccccccc}
\hline \multicolumn{1}{c}{ PROBLEMAS } & \multicolumn{10}{c}{ CARACTERISTICAS TD } \\
\multicolumn{1}{c}{ AMBIENTALES } & TAM & ORG & TEN & OCU & EDA & EDU & PAR & CAP & ATEC \\
\hline Cambios del clima & 0,0 & $-0,1$ & 0,1 & $-0,2$ & 0,1 & 0,0 & 0,1 & 0,3 & 0,1 \\
Erosión & 0,1 & 0,1 & 0,0 & $-0,1$ & 0,1 & 0,0 & 0,2 & 0,0 & 0,1 \\
Cantidad/calidad del agua & 0,2 & 0,0 & 0,0 & $-0,2$ & 0,2 & $-0,1$ & 0,1 & 0,2 & 0,3 \\
Deslizamientos & 0,0 & 0,4 & 0,2 & 0,1 & 0,0 & 0,1 & $-0,1$ & 0,0 & 0,0 \\
Deforestación & 0,1 & $-0,1$ & $-0,1$ & $-0,2$ & 0,0 & 0,1 & $-0,2$ & 0,1 & 0,2 \\
Pérdida de biodiversidad & 0,0 & 0,2 & $-0,1$ & 0,0 & 0,1 & $-0,1$ & $-0,3$ & 0,0 & $-0,2$ \\
\hline TAM: Número de animales en el predio que maneja, ORG: Origen, TEN: Tenencia del predio, OCU: Ocupa el \\
predio, EDA: Edad, EDU: Educación, PAR: Participación en organizaciones, CAP: Capacitaciones recibidas y \\
ATEC: Asistencia técnica recibida.
\end{tabular}

Relación entre características de los tomadores de decisión y el uso de prácticas de conservación del agua

Este análisis tampoco presenta resultados significativos, sin embargo, la tendencia de los signos muestra relaciones directas entre el uso de las prácticas de conservación y algunas características de los tomadores de decisión como el tamaño $(+)$, el origen $(+)$, la tenencia $(+)$, el nivel de educación $(+)$, la participación $(+)$ y la asistencia técnica $(+)$. Con la característica de ocupación, que representa si el tomador de decisiones 
habita o no en el predio, la relación con todas las prácticas es inversa (-) (Cuadro 7). Esto último podría significar que los tomadores de decisión que habitan en el predio tienden a no usar prácticas de conservación, quizás por no ser los dueños de los predios.

Cuadro 7. Coeficientes de Correlación entre prácticas de conservación utilizadas y características de los tomadores de decisión de los predios ganaderos de la cuenca.

\begin{tabular}{lccccccccc}
\hline \multicolumn{1}{c}{ PRACTICAS DE } & \multicolumn{10}{c}{ CARACTERISTICAS TD } \\
CONSERVACIÓN & TAM & ORG & TEN & OCU & EDA & EDU & PAR & CAP & ATEC \\
\hline Prácticas Tipo 1 (n: 41) & 0,2 & 0,3 & 0,2 & $-0,2$ & $-0,2$ & 0,4 & 0,1 & 0,0 & 0,5 \\
Prácticas Tipo 2 (n: 4) & 0,1 & 0,2 & 0,1 & $-0,2$ & $-0,1$ & 0,3 & 0,0 & 0,2 & 0,4 \\
Prácticas Tipo 3 (n: 45) & 0,3 & 0,2 & 0,3 & $-0,4$ & 0,1 & 0,3 & 0,0 & $-0,2$ & 0,3 \\
\hline
\end{tabular}

Tipo 1: Uso más reducido del agua, Tipo 2: Disminución del agotamiento y Tipo 3: Mayor r reposición del agua. TAM: Número de animales en el predio que maneja, ORG: Origen, TEN: Tenencia del predio, OCU: Ocupa el predio, EDA: Edad, EDU: Educación, PAR: Participación en organizaciones, CAP: Capacitaciones recibidas y ATEC: Asistencia técnica recibida. n: total de predios donde se implementan las prácticas.

Para las prácticas Tipo 1 las mayores correlaciones se dan con características como la asistencia técnica $(C C: 0,5)$ y el nivel de educación de los tomadores de decisión (CC: 0,4). Para las prácticas Tipo 2 la mayor correlación se presenta con la asistencia técnica (CC: 0,4$)$, mientras que para las prácticas Tipo 3 la mayor correlación se da de manera inversa con la ocupación (CC: -0,4) (Cuadro 7). Estos resultados sugieren que los diferentes tipos de prácticas se relacionan de manera particular con características de los tomadores de decisión, por ejemplo, las prácticas Tipo 1 tienen más relación con el uso de asistencia técnica y el nivel educativo del tomador de decisiones, mientras que las prácticas Tipo 2 están más relacionadas con la asistencia técnica.

\section{CONCLUSIONES}

Los sistemas de producción agrícola y ganadero son fuente de ingreso y generación de empleo en las zonas rurales, pero generan impactos ambientales importantes si no se manejan adecuadamente. Dado que la gestión de dichos sistemas depende, en primer lugar, de los tomadores de decisión en los predios, es fundamental conocer sus características, la percepción que tienen sobre los problemas ambientales de sus predios y las prácticas que realizan para mitigar los impactos ambientales generados por sus actividades o para conservar los recursos naturales.

En la cuenca alta del río Guarinó, zona eminentemente ganadera, los ganaderos se caracterizan por su heterogeneidad, en cuanto a edad, educación y tamaño de los predios. Mientras que en género, tenencia de los predios, origen, participación en organizaciones y permanencia en los predios hay similitud. En cuanto a lo primero, se destaca un nivel de educación relativamente alto y predios de tamaño medio, lo que daría pautas para el diseño y puesta en ejecución de iniciativas que propendan por la adopción de prácticas que contribuyan a la conservación de los recursos naturales. En relación con lo segundo, es importante considerar estas características en programas de capacitación, sobre todo, en la característica de ausentismo de los predios. 
Con las evidencias aportadas por este trabajo, no es posible identificar las causas de la escasa capacitación y del poco uso de asistencia técnica, pero se presume que puedan estar relacionadas con el ausentismo de los propietarios, o quizás, por falta de reconocimiento de la importancia que estas tienen para el mejoramiento de los sistemas de producción, lo cual debería considerarse en las propuestas de capacitación y programas de asistencia técnica que quieran implementar instituciones como la CAR o el Fondo Ganadero de Caldas en la zona.

En cuanto a la percepción que tienen los tomadores de decisión en la zona, hay una mayor percepción de los cambios en el clima y el problema que menos se percibe es la pérdida de la biodiversidad. La diferencia en esta percepción puede obedecer a que los cambios en el clima afectan directamente sus prácticas, actividades y recursos. Por ejemplo, lluvias torrenciales producen erosión y deslizamientos, sequias afectan las fuentes de agua, heladas dañan las pasturas. En tanto que la biodiversidad es algo que los productores no perciben, pues no afecta directamente sus actividades.

De ahí que en la mayoría de los predios ganaderos de la cuenca se implementa alguna práctica de conservación del agua. $Y$ entre ellas, las más utilizadas en la zona son las relacionadas con reposición del agua. Esta situación ratifica lo dicho antes. Y sobre todo, en un recurso tan vital para la actividad ganadera como es el agua. Por ello, se requiere un trabajo adicional para que los tomadores de decisión tengan mejores elementos para mejorar la percepción de problemas ambientales que no son tan evidentes, como son la biodiversidad, la contaminación de las aguas o la pérdida de la fertilidad del suelo.

En cuanto a la relación que existe entre características de los tomadores de decisión, los problemas ambientales que perciben y las prácticas de conservación que implementan, no hay relación significativa, sin embargo, para algunas características se evidencian relaciones directas, es decir, que la percepción y la puesta en práctica se relacionan positivamente.

Para determinar cómo las características de los tomadores de decisión influyen sobre la percepción de los problemas y las prácticas utilizadas por los tomadores de decisión en los predios, se requiere realizar análisis más finos. Tal vez, utilizar metodologías participativas que permitan indagar aspectos que los métodos convencionales como la encuesta no permiten captar.

\section{RECOMENDACIONES}

De lo anterior, surgen algunas ideas que se deben tener en cuenta en el momento de realizar intervenciones, acciones o proyectos dirigidos a la conservación de la cuenca alta del río Guarinó. Entre ellas, se debe considerar:

- Reconocer las particularidades de los tomadores de decisión de la zona y las variaciones que estos presentan principalmente en cuanto al tamaño de los predios que manejan, la edad y nivel educativo que tienen.

- Involucrar a las organizaciones presentes en la zona, particularmente la Cooperativa Ovina, donde participan la mayoría de los tomadores de decisión. Se trata de utilizar estrategias más eficientes en términos de los objetivos de los proyectos implementados en la región para conservar la cuenca. 
- Tener presente que las acciones dirigidas a solucionar los problemas de erosión y mejorar la calidad/cantidad del agua pueden tener buena aceptación en la zona, ya que estos problemas son los problemas más percibidos; además, de que estos tomadores de decisión son propietarios de la tierra y eso tiene que ser bien aprovechado.

- Reconocer que las acciones de conservación de la biodiversidad en la cuenca necesitan una previa sensibilización y educación de los tomadores de decisión, ya que este problema casi no es percibido por ellos.

- Valorar la calidad de las prácticas que se implementan en los predios, para orientar las acciones en las unidades de producción o en la región, de manera que se garantice la eficiencia en los programas de conservación emprendidos.

- Realizar estudios más específicos que permitan identificar y confirmar las relaciones que pueden existir entre las características de los tomadores de decisión, los problemas ambientales que perciben y las prácticas de conservación que usan.

\section{BIBLIOGRAFÍA}

- Barber, J. S., Biddlecom, A. E., y Axinn, W. (2003). Neighborhood Social Change and Perceptions of Environmental Degradation. Population \& Environment, 25(2), 77-108. Desde: http://deepblue.lib.umich.edu/bitstream/2027.42/43500/1/11111_2 004_Article_479782.pdf

- Berenson, M. L., y Levine, D. M. (1991). Estadística para administración y economía: conceptos y aplicaciones. México: McGraw-hill.

- Delgado, L. E., Bachmann, P. L., y Oñate, B. (2007). Gobernanza ambiental: Una estrategia orientada al desarrollo sustentable local a través de la participación ciudadana. Revista Ambiente y Desarrollo de CIPMA, 23(3), 68-73. Desde: http://www.ecomanage.info/products/2007_Delgado_etal_RAD.p df

- Corporación Autónoma Regional de Caldas -CORPOCALDAS(2001). Agenda para la gestión ambiental del municipio de Marulanda. Manizales, Colombia: CORPOCALDAS.

- Federación Nacional de Ganaderos de Colombia -FEDEGAN-. (2006). Plan Estratégico de la Ganadería Colombiana 2019. Bogotá, D.C.: San Martín Obregón \& Cía. Desde: http://portal.fedegan.org.co/Documentos/pega_2019.pdf

- Gil, H. A., De Prada, J. D., Hernández, J., Pereyra, C., y Angeli, A. R. (2008). Análisis de la Percepción del Productor sobre la Problemática Ambiental. En De Prada, J. D., y Penna, J. (eds.), Percepción económica y visión de los productores agropecuarios de los problemas ambientales en el Sur de Córdoba, Argentina. Estudios socioeconómicos de la sustentabilidad de los sistemas de producción y recursos naturales, No. 8. Buenos Aires: Ediciones Instituto Nacional de Tecnología Agropecuarias INTA-. Obtenido el 15 de septiembre de 2010, desde http://www.inta.gov.ar/ies/docs/docs_area/pdfs/percepcion_IES_ 08.pdf

- Murgueitio, E. (1999). Reconversión ambiental y social de la ganadería bovina en Colombia. Desde: http://www.agronet.gov.co/www/docs_si2/20061127114225_Reco nversion\%20ambiental\%20social\%20de\%20ganaderia\%20en $\% 20$ Colombia.pdf 

Desde: http://www.Irrd.org/lrrd15/10/murg1510.htm

- Murgueitio, E., Arango, H. A., Calle, Z., Naranjo, J. F., Cuartas, C. A., y Caro, M. F. (2008). Recurso natural agua. En Medidas integrales para el manejo ambiental de la ganadería bovina. Colombia: FEDEGAN, SENA y CIPAV.

- Nieto, M. I., Riedel, J. L., y Vera, G. T. (2002). Percepciones y actitudes de pequeños productores de la región de los llanos de la Rioja, Argentina, sobre prácticas agrícolas de secano (chacras). Revista de Desarrollo rural y Cooperativismo Agrario, 6, 193-204.

- Organización de las Naciones Unidas para la Agricultura y la Alimentación -FAO-. (2007). Cómo enfrentarse a la interacción ganadería medio ambiente. Documento Comité de Agricultura. Roma (IT).

Desde: ftp://ftp.fao.org/docrep/fao/meeting/011/j9420s.pdf

- Parques Nacionales Naturales de Colombia. (s.f.). Bosque Andino o niebla. Obtenido el 16 de septiembre de 2010, desde http://www.parquesnacionales.gov.co/PNN/portel/libreria/php/de cide. php? patron $=01.201203$

- Steinfeld, H., Gerber, P., Wassenaar, T., Castel, V., Rosales, M. y de Haan, C. (2006). Livestock's long shadow: Environmental issues and options. Roma, Italia: FAO. Desde: http://www.fao.org/docrep/010/a0701e/a0701e00.HTM

1. MVZ, Mg. en Desarrollo Rural. Profesora Universidad de Caldas. E-mail: marcela.patino@ucaldas.edu.co

2. IA, Doctor en Sociología del Medio Ambiente. Profesor Universidad de Caldas. E-mail: isaias.tobsura@ucaldas.edu.co

3. Tomadores de decisión se refiere a aquellos cuyas acciones dependen de o afectan los servicios ecosistémicos. Los tomadores de decisión pueden trabajar a diferentes escalas (locales, provinciales, nacionales o internacionales) para lograr el desarrollo de objetivos a través de políticas, planes o proyectos, o para focalizar planes de conservación, manejo de recursos naturales y protección ambiental.

4. Para el análisis de las características de los tomadores de decisión (género, nivel de escolaridad, edad, etc.) en los casos en que son dos los tomadores de decisión del predio, se tomaron como referencia los datos de los hijos, en el caso de propietarios e hijos tomando decisiones, y los datos de los propietarios, en el caso de propietario y administradores tomando decisiones. 